\title{
Analyse Conceptuelle Et Optimisation De La Fiabilité Des Systèmes : Cas D'une Unité De Production De Farines Des Nourrissons
}

\begin{abstract}
Aslain Brisco Ngnassi Djami
PhD Candidate, National Advanced School of Agro - Industrial Sciences, Ngaoundere University, Ngaoundere, Cameroon

Wolfgang Nzie

Département des Sciences et Techniques de Base pour l'Ingénieur, EGCIM, Université de Ngaoundéré, Ngaoundéré, Cameroun

Doi: 10.19044/esj.2018.v14n24p283 URL:http://dx.doi.org/10.19044/esj.2018.v14n24p283

Abstract

The purpose of this article is to propose an approach to analyse and optimize the topology of reliability in the design of mechanical systems. The application is made on a chain of production of infant flours. Indeed, the systems that we design are more and more complex and must be realized at reduced costs. These systems are "multi-domains" because many trades come into play. High reliability for complex topology systems is required. Thus, we present a methodology for the analysis of reliability in the design process that groups models based on diagrams. Finally, to optimize the topological configuration and the reliability of the system, the so-called "robust design" method coupled with an appropriate algorithm is deployed. The expected result is: the proposal of a methodology for optimizing the topology and the reliability of the systems.
\end{abstract}

Keywords: Reliability, Robust Design, Optimization, Flours, Infant.

\section{Résumé}

Le but de cet article est de proposer une démarche d'analyse et d'optimisation de la topologie de la fiabilité dans la conception des systèmes mécaniques. L'application est faite sur une chaine de production de farines des nourrissons. En effet, les systèmes que l'on conçoit sont de plus en plus complexes et doivent être réalisés à des coûts réduits. Ces systèmes sont «multi-domaines», car plusieurs corps de métiers entrent en jeu. Une fiabilité élevée pour ces systèmes de topologie complexe s'impose. Ainsi, nous présentons une méthodologie pour l'analyse de la fiabilité dans le processus de conception qui regroupe des modèles basés sur des diagrammes. Enfin, 
pour optimiser la configuration topologique et la fiabilité du système, la méthode dite de « conception robuste » couplée avec un algorithme approprié est déployée. Le résultat attendu est: la proposition d'une méthodologie d'optimisation de la topologie et de la fiabilité des systèmes.

Mots clés : Fiabilité, Conception robuste, Optimisation, Farines, Nourrissons.

\section{Introduction}

La fiabilité est définie comme la probabilité qu'un système ou un composant effectue une fonction requise dans des conditions de fonctionnement indiquées sans échec, pour une période de temps donnée (Mohammad, 2016). La fiabilité est donc :

$$
R(T \succ t)=e^{-\int_{0}^{t} \lambda(u) d u}
$$

où $\mathrm{R}(\mathrm{t})$ et $\lambda(t)$ désignent respectivement la fiabilité et le taux de défaillance à l'instant $t$.

De nos jours, la fiabilité est un facteur indispensable dans la conception et la fabrication de produits, car dans les industries sensibles telles que celles de production de farines des nourrissons, tout échec dans l'exécution des systèmes peut entraîner des dommages irréparables. Une méthodologie pour l'analyse de la fiabilité dans le processus de conception intégrant des modèles basés sur des diagrammes (AMDEC, $\mathrm{AdD}$, etc) a été proposé à cet effet par (Bhamare et Rathore, 2007).

L'augmentation de la complexité d'un système mécanique, fait diminuer sa fiabilité, si l'on ne prend pas des mesures compensatoires. Un système formé de composants indépendants, en série est tel que, si un seul des composants est en panne, le système ne fonctionne plus (Beleulmi et al. 2011). On dit que le système est sans redondance. La fiabilité de ces systèmes se détériore dramatiquement avec l'augmentation du nombre des composants. Pour optimiser la fiabilité du système, nous déploierons la méthode de «conception robuste» couplée avec un algorithme, tout en ayant au préalable faite une analyse de la fiabilité de notre unité, dans le but de maximiser la fiabilité du système sous contraintes de couts afin d'obtenir une meilleure redondance.

\section{Revue De La Littérature \\ Prise en compte de la fiabilité en conception}

Les systèmes que l'on conçoit sont de plus en plus complexes et doivent être réalisés à des coûts et en un temps qui sont de plus en plus restreints (Gittard, 2009). Ces systèmes comme les unités de production de farine, sont «multi-domaines »; plusieurs corps de métiers vont rentrer en jeu lors de la conception. Une fiabilité élevée pour ces systèmes complexes est 
demandée. Pour pouvoir atteindre cet objectif, la fiabilité doit être prise en compte dès les phases amont de la conception. De nombreuses méthodes sont connues (tels que les AMDEC, les arbres de défaillances,...) pour la réalisation d'analyse de la fiabilité (Gittard, 2009).

Deux approches sont possibles pour observer le comportement du système (Ozouf et Pillet, 2006) :

- La boite noire (ou analyse fonctionnelle externe), qui correspond à l'aspect externe uniquement, en ignorant la constitution du système pour ne considérer que ses entrées / sorties et les effets de son action sur l'environnement. Cette approche conduit à l'obtention des fonctions de services (F.S).

- La boite blanche (ou analyse fonctionnelle interne), qui correspond à l'aspect interne seulement, en regardant l'ensemble des éléments en interaction mutuelle pour mettre en évidence le fonctionnement du système. Cette approche conduit à l'obtention des solutions technologiques (F.T).

Des outils pertinents d'analyse de la fiabilité, comme énoncé plus haut, nous avons entre autres :

- Les Arbres de Défaillances (AdD), qui ont pour objectif d'analyser et de réduire les risques. Ce sont des outils de recensement de toutes les causes et défauts entrainant l'apparition d'un évènement indésirable. Au même titre que le comportement d'un système dépend de ses composants, le comportement du composant ou du sous-système dépend de ses différents modes de défaillance (Zwingmann, 2005). Cette technique est dépendante des probabilités d'occurrence des évènements.

- L'Analyse des Modes de Défaillances, de leurs Effets et de leur Criticité (A.M.D.E.C), qui est avant tout une méthode d'analyse des systèmes, s'appuyant sur un raisonnement inductif (cause-conséquences), pour l'étude organisée des causes, des effets des défaillances et de leur criticité (Ozouf et Pillet, 2006).

\section{Méthodes de conception robustes}

Filiol (2010) présente trois méthodes de conception robuste, décrites comme suit :

\section{* Optimisation du rendement}

Les méthodes d'optimisation du rendement ont pour but de déterminer les paramètres de conception qui maximisent le rendement paramétrique. On en distingue des méthodes statistiques, dont le but est l'évaluation du rendement qui est effectuée par un échantillonnage au moyen des algorithmes déterministes non-linéaires de Keramat et Kielbasa (1997) ; Soin et Spence (1980) et des méthodes déterministes, dont le but est l'optimisation indirecte du rendement. Ici on part du fait que le rendement s'écrit comme l'intégrale d'une densité de probabilité et donc optimiser le rendement revient juste à 
optimiser le volume qu'occuperait cette densité dans une certaine région dite d'acceptation ou de tolérance (Director et al.1992).

\section{* Optimisation robuste}

Le problème de conception robuste, peut s'exprimer sous la forme d'un problème d'optimisation dont la résolution conduit à un jeu de valeurs pour les paramètres de conception qui minimise une certaine fonction de coût f et qui garantit que les spécifications sur des performances $g_{1}$ sont respectées pour toutes les dispersions paramétriques appartenant à la région de tolérance $\mathrm{RT}_{\Delta \mathrm{T}}$. Le problème de conception robuste peut donc s'écrire sous la forme générale suivante :

$$
\begin{array}{cl}
\text { minimiser } & \mathrm{f}(\mathrm{C}, \Delta \mathrm{T}) \\
\text { tel que } & g_{\ell}(C, \Delta T) \prec \mathrm{O}, \ell \in\{1, \ldots, p\} \\
& \mathrm{C} \in D \\
& \forall \Delta T \in R T_{\Delta T}
\end{array}
$$

- C est le vecteur des paramètres de conception appartenant au domaine de conception D ;

- $\Delta \mathrm{T}$ est le vecteur des variations technologiques appartenant à la région de tolérance $\mathrm{RT}_{\Delta \mathrm{T}}$;

- f est la fonction de coût à minimiser ;

- $g_{\mathrm{l}}$ sont les performances qui doivent respecter les spécifications définies par des inégalités.

La difficulté majeure dans ce problème d'optimisation est liée à la contrainte « $\Delta \mathrm{T} \in \mathrm{RT}_{\Delta \mathrm{T}}$ », c'est-à-dire « quelles que soient les fluctuations paramétriques $\Delta \mathrm{T}$ appartenant à la région de tolérance $\mathrm{RT}_{\Delta \mathrm{T}} \gg$ dans la formulation du problème de conception robuste. La région de tolérance $\mathrm{RT}_{\Delta \mathrm{T}}$ étant un ensemble infini, la contrainte $« \forall \Delta \mathrm{T} \in \mathrm{RT}_{\Delta \mathrm{T}} »$, peut donc être vue comme une infinité de contraintes qui doivent être vérifiées. Une solution pour s'affranchir de cette difficulté consiste à reformuler les contraintes en faisant intervenir la borne supérieure des performances (Mukherjee, 2000).

\section{* Optimisation par des indices de capabilité}

Une dernière approche indirecte pour traiter la conception robuste est dérivée de la méthode d'amélioration de la qualité mise au point par $\mathrm{G}$. Taguchi. Cette approche est fondée sur deux critères de mesure de la variabilité appelés indices de capabilité (Aftab and Styblinski, 1994). En combinant ces indices, on peut définir pour chaque performance une mesure de la robustesse. L'intérêt principal de cette méthode est de pouvoir contrôler 
indépendamment la variabilité des performances et leur centrage (Aftab and Styblinski, 1994); (Debyser and Gielen, 1998). Cependant, l'utilisation des indices de capabilité est fondée sur l'hypothèse de normalité des performances, qui est loin d'être vérifiée en pratique. L'objectif de ce papier est donc de proposer une démarche d'analyse et déployer un outil d'optimisation de la fiabilité dans la conception des systèmes mécaniques tels que ceux de production de farines des nourrissons.

\section{Méthodes}

L'analyse fonctionnelle permet de structurer la recherche de la satisfaction du client en représentant le produit du point de vue du « service rendu », et encourage la créativité en laissant ouvert le choix des solutions techniques (Mathelin, 2006).

Nous utilisons donc pour concevoir et analyser la fiabilité du produit, l'analyse fonctionnelle avec comme principaux outils :

- La bête à cornes, qui permet d'exprimer la recherche du besoin ;

- Le diagramme pieuvre, qui permet de définir les liens (c'est-à-dire les fonctions de service) entre le système et son environnement. Ce diagramme permet de recenser la plupart des fonctions du système ;

- Le diagramme FAST, qui permet la recherche de solutions technologiques.

Pour l'optimisation de la fiabilité, la méthode de conception dite « robuste 》 couplée à un algorithme sera déployée.

Une architecture de type parallèle - série a été choisie car elle conduit à une bonne fiabilité.

La réalisation des systèmes devant remplir des missions dans des conditions interdisant toute opération d'entretien ou maintenance (vol d'avion, satellites artificiels, fusée, etc.) nécessite une fiabilité maximale pendant la durée de la mission. Il faudra de plus tenir compte de certaines contraintes et natures techniques (volume, poids, encombrement) ou économiques (coûts des composants, budget alloué) (Gondran et al.1995).

Ainsi on pourra trouver dans le Cahier des charges des spécifications telles que $\ll$ le dispositif devra avoir sur un temps $\mathrm{T}$ une fiabilité maximale, son coût restant inférieur à un coût $\mathrm{C}$ et son poids restant inférieur à $\mathrm{P} \gg$, ou bien

《la fiabilité du système devra être sur une période $T$ supérieure à $\mathrm{R}_{\min }$, son coût devant être le plus bas possible $\gg$.

On se limitera ici au cas d'un système à configuration parallèle-série, c'est-à-dire constitué de K étages devant tous fonctionner en série pour que le système puisse fonctionner. 


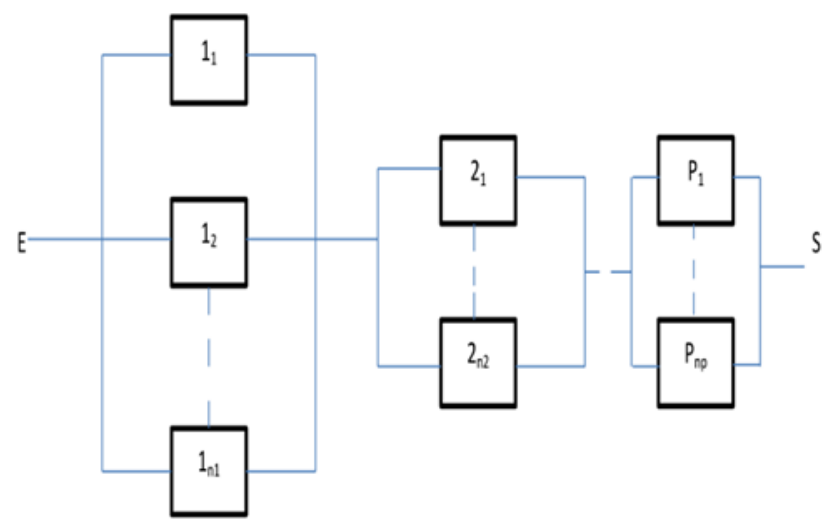

Fig.1 Diagramme de fiabilité d'une configuration parallèle-série.

La fiabilité $\mathrm{R}$ du système sur une période donnée est le produit de la fiabilité des étages :

$$
R=\prod_{i=1, K} R_{i}
$$

L'étage i sera composé de $n_{i}$ éléments distincts ayant chacun pour fiabilité $\mathrm{p}_{\mathrm{i}}$. On a donc :

$$
R_{i}=1-\prod_{l=1, n}\left(1-p_{i}\right)
$$

Si à chaque type d'éléments $i$ on associe des coûts $C_{i j}(j=1,2, \ldots, m)$ (poids, volume, etc.) les contraintes technologiques et économiques pourront s'écrire :

$$
\sum_{i=1, K}^{n} C_{i j} n_{i} \leq C_{j} \quad \forall j=1,2, \ldots, m
$$

Le problème du choix de la recherche de la redondance maximale revient donc à déterminer un vecteur $\mathrm{n}=\left(\mathrm{n}_{1}, \mathrm{n}_{2}, \ldots, \mathrm{n}_{\mathrm{k}}\right)$ avec $\mathrm{n}_{\mathrm{i}}$ entiers positifs, solution du problème :

$$
\left\{\begin{aligned}
& \operatorname{maximiser} \mathrm{R}(\mathrm{n})= \prod_{\mathrm{i}=1, \mathrm{~K}}^{\mathrm{n}}\left[1-\prod_{l=1, n}\left(1-p_{i}\right)\right] \\
& a \mathrm{vec} \\
& \text { sous les contraintes } \sum_{i=1, K}^{n} C_{i j} n_{i} \leq C_{j} \quad \forall j=1,2, \ldots, m \\
& n_{i} \text { entier positif } \quad \forall i=1,2, \ldots, K
\end{aligned}\right.
$$

La solution de ce problème est en général très difficile car la fonction $R(n)$ est non linéaire et les solutions recherchées $n_{i}$ sont entières. 


\section{Résultats}

Dans cette partie du papier, nous présentons des résultats obtenus suite à la démarche énoncée dans la partie méthodes. Compte tenu de la complexité du système d'étude, nous avons restreint nos résultats à l'étude d'un des systèmes importants dans la production de farines des nourrissons : le broyeur à marteaux.

\section{Enoncé du besoin par l'outil bête à cornes}

Enoncer le besoin revient à exprimer l'exigence fondamentale qui justifie la conception du système. Cette approche est fondée sur la dématérialisation du système, c'est-à-dire sur la considération exclusive des satisfactions qu'il offre afin de combler le besoin latent du demandeur. Pour cela, on est amené à se poser les trois questions essentielles à savoir :

A qui, à quoi le système rend-il service ? Sur qui, sur quoi agit-il ? Dans quel but ? L'outil de représentation dit «bête à cornes » permet d'y répondre. Il est schématisé à la Figure 2 ci-contre :

A qui rend service le produit?

Sur quoi agit le système?

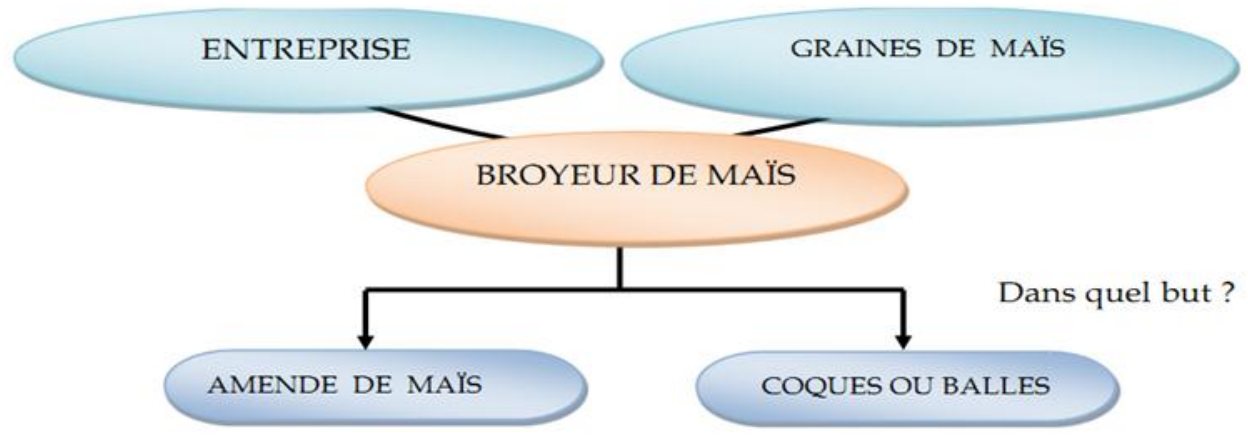

Fig.2 Diagramme Bêtes à cornes du broyeur de Maïs.

Table 1 : Contrôle de validation du besoin.

\begin{tabular}{|c|c|}
\hline Questions & Réponses \\
\hline $\begin{array}{l}\text { Pourquoi le besoin existe-t-il ? } \\
\text { - Dans quel but? } \\
\text { - Pour quelle(s) raison(s) ? }\end{array}$ & $\begin{array}{l}\text { - Broyage des grains de maïs ; } \\
\text { - Perception d'un nouveau marché : } \\
\text { exportation, vente et offre de service. }\end{array}$ \\
\hline $\begin{array}{l}\text { Qu'est ce qui pourrait faire disparaitre le } \\
\text { produit? }\end{array}$ & $\begin{array}{l}\text { La mise sur pied d'une unité automatisée de } \\
\text { production de broyat pour production de farines } \\
\text { des nourrissons. }\end{array}$ \\
\hline Y'a-t-il risque de voir disparaitre le besoin ? & $\begin{array}{l}\text { La diversité de céréales et la production annuelle } \\
\text { sans cesse croissante. }\end{array}$ \\
\hline
\end{tabular}

\section{Identification des fonctions par le diagramme Pieuvre}

L'identification des fonctions est très importante dans une démarche de conception. En effet, les fonctions reflètent d'une part la description du besoin à remplir par le système et d'autre part, elles donnent des pistes d'orientation 
sur le choix des différents scénarios à analyser. Le système est donc considéré ici comme un agencement de fonctions et non comme un assemblage de solutions.

Pour définir les fonctions de notre système, nous utilisons l'outil de représentation dit « diagramme pieuvre ».

Ce diagramme représente le système entouré des éléments extérieurs en contact avec lui. Chaque fois que le système permet de mettre en relation deux éléments du milieu extérieur, il y a service rendu. Donc, en prenant tous les éléments du milieu extérieur 2 par $\mathbf{2}$, chaque fois qu'il est possible d'exprimer un service rendu, il y a fonction principale (FP). Chaque fois qu'un élément du milieu extérieur exerce ou subit une action du système, il y a une fonction de contrainte (FC).

Connaissant le besoin précédemment énoncé par l'outil bête à cornes et tenant compte des spécifications de l'objectif de conception, nous construisons le diagramme pieuvre du broyeur.

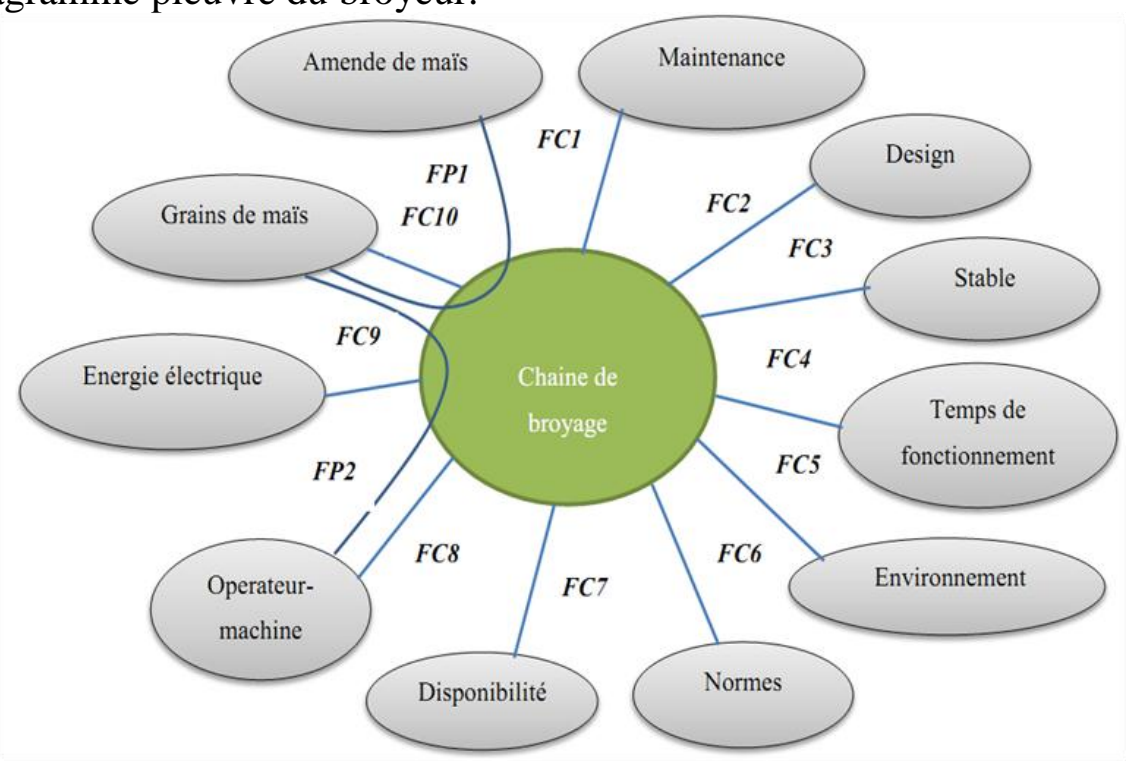

Fig.3 Graphe des interactions de la chaine de broyage.

Les caractéristiques des fonctions de service sont données dans le tableau cidessous :

Table 2 : Caractéristiques des fonctions de service.

\begin{tabular}{|c|c|c|}
\hline $\begin{array}{c}\text { Fonction d'usage/ fonction } \\
\text { d'estime }\end{array}$ & Symboles & Description \\
\hline \multirow{2}{*}{ Fonction principale } & FP1 & $\begin{array}{c}\text { Réduire des grains de maïs pour } \\
\text { en faire des amendes }\end{array}$ \\
\cline { 2 - 3 } & FP2 & $\begin{array}{c}\text { Introduire et ordonne le transport } \\
\text { des grains de maïs }\end{array}$ \\
\hline & FC1 & Etre facilement maintenable \\
\hline
\end{tabular}




\begin{tabular}{|c|c|c|}
\hline \multirow{4}{*}{ Fonctions contraintes } & FC2 & Plaire à l'utilisateur \\
\cline { 2 - 3 } & FC3 & Etre stable \\
\cline { 2 - 3 } & FC4 & $\begin{array}{c}\text { Avoir une durée de } \\
\text { fonctionnement maximale (12h) }\end{array}$ \\
\cline { 2 - 3 } & FC5 & $\begin{array}{c}\text { S'adapter à l'environnement } \\
\text { sociotechnique }\end{array}$ \\
\cline { 2 - 3 } & FC6 & Respecter les normes \\
\cline { 2 - 3 } & FC7 & Utiliser les matériaux locaux \\
\cline { 2 - 3 } & FC8 & Respecter les normes \\
\cline { 2 - 3 } & FC9 & Utiliser de l'énergie électrique \\
\cline { 2 - 3 } & FC10 & Utiliser les grains de maïs \\
\hline
\end{tabular}

\section{Diagramme FATST du broyeur de maïs}

- Cadre d'utilisation : Production industrielle ;

- Fonction globale d'usage : Broyer 3.5 tonnes/heure ;

- Mode de fonctionnement: Manuelle.

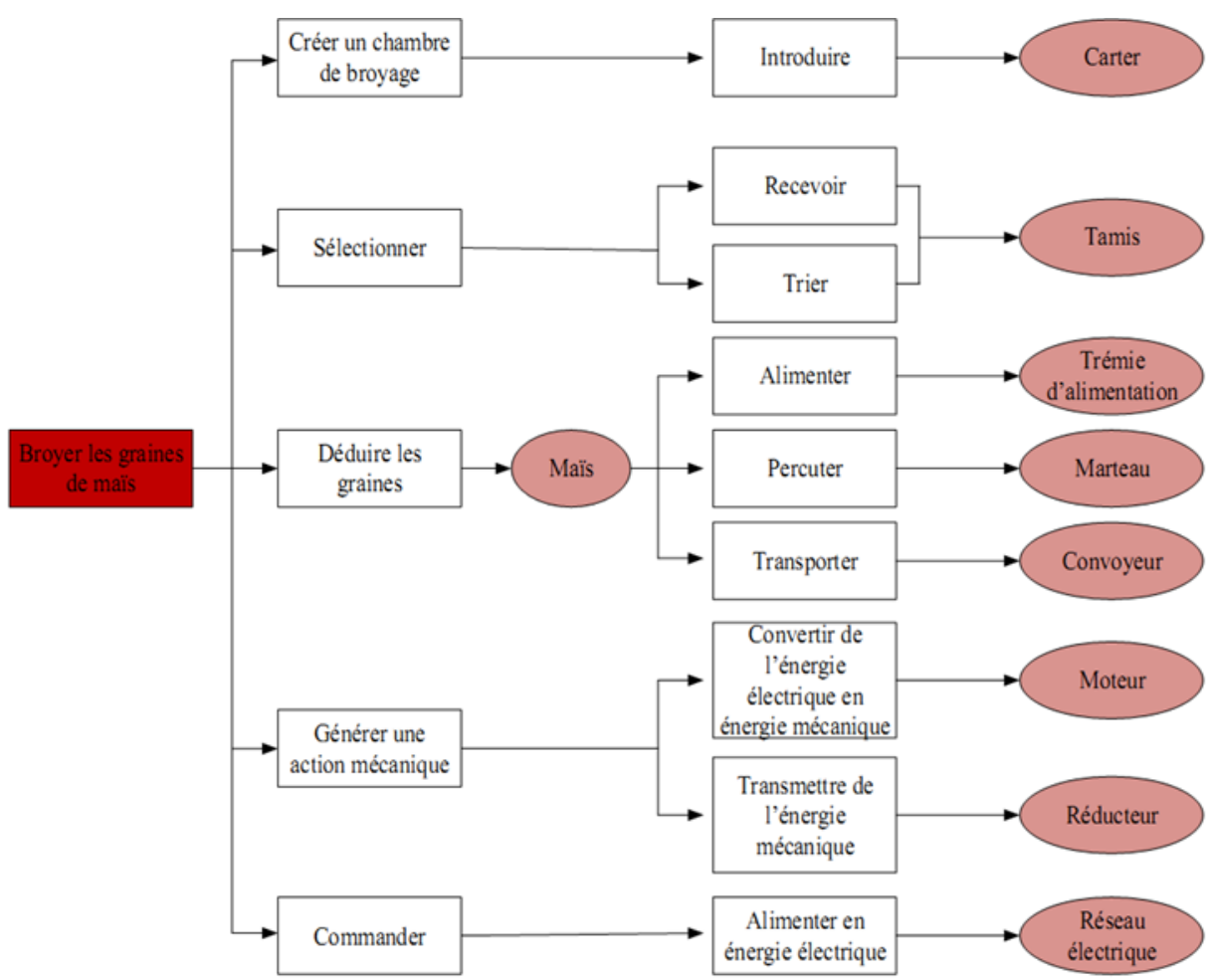

Fig.4 : Diagramme FAST du broyeur des graines de Maïs

\section{Etude de cas d'optimisation de la fiabilité}

Nous allons considérer notre broyeur de farines des nourrissons, constitué de plusieurs ensembles (convoyeur, trémie d'alimentation, broyeur, et autre) devant fonctionner dans un système de redondance parallèle - série. 
Ces opérations unitaires, constitué de $\mathbf{N}$ éléments ( $\mathbf{N}$ fixé) sont disposées dans $\mathbf{K}$ étages ( $\mathbf{K}$ fixé) d'éléments en parallèle qui doivent fonctionner simultanément en série. Notre objectif ici étant de maximiser la fiabilité sous contrainte de Coûts afin d'obtenir une meilleure redondance. On déterminera donc le nombre de composants $n_{i}$ à ajouter sur l'étage $i$ afin d'élever sa fiabilité, et donc la fiabilité globale du système.

On suppose ici qu'il n'ya qu'une contrainte (par exemple le volume). Le problème de maximisation de la fiabilité pour un nombre d'éléments $\mathbf{N}$ fixé s'écrit donc :

$$
\left\{\begin{array}{c}
\max \mathrm{R}(\mathrm{n})=\prod_{\mathrm{i}=1, \mathrm{~K}}^{\mathrm{n}}\left[1-\prod_{l=1, n}\left(1-p_{i}\right)\right] \\
\operatorname{avec} \sum_{i=1, K}^{n} n_{i}=N \\
n_{i} \text { entier } \geq 0
\end{array}\right.
$$

En supposant $\mathrm{p}_{\mathrm{i}}$, fiabilité d'un élément de l'étage $\mathrm{i}$, grand, $\mathrm{q}_{\mathrm{i}}=1-\mathrm{p}_{\mathrm{i}}$, est petit et $\mathrm{R}(\mathrm{n})$ peut s'écrire : $R(n)=1-\sum_{i=1, K}^{n}\left[\prod_{l=1, n} q_{i}\right]$

Nous prendrons cette formule dans la suite pour pouvoir approcher la solution optimale simplement.

L'algorithme suivant donne alors la solution optimale du problème.

\section{Algorithme}

1. ( N donné ). Pour i de 1 à $\mathrm{K}, \mathrm{n}_{\mathrm{i}}=0$

$$
R=1-\sum_{i=1, K}^{n}\left[\prod_{I=1, n} q_{i}\right]
$$

2.Choisir $\mathrm{i}_{0}$ tel que

$\mathrm{r}=-\prod_{\mathrm{l}=1, \mathrm{n}+2} \mathrm{q}_{\mathrm{i}_{0}}+\prod_{1=1, \mathrm{n}+1} \mathrm{q}_{\mathrm{i}_{0}}=\max \left(-\prod_{1=1, \mathrm{n}+2} \mathrm{q}_{\mathrm{i}}+\prod_{1=1, \mathrm{n}+1} \mathrm{q}_{\mathrm{i}}\right)$

3. Faire $\mathrm{n}_{\mathrm{i}_{0}} \leftarrow \mathrm{n}_{\mathrm{i}_{0}}+1, \mathrm{R} \leftarrow \mathrm{R}+\mathrm{r}$

$$
\mathrm{N} \leftarrow \mathrm{N}-1
$$

$\mathrm{Si} \mathrm{N}=0$, FIN, sinon aller en 2 .

La solution $\mathrm{n}^{*}$ obtenue à la fin de l'algorithme est optimale. 


\section{Conclusion}

Parvenu au terme de la rédaction de ce papier dont l'intitulé est «Analyse conceptuelle et optimisation de la fiabilité des systèmes : cas d'une unité de production de farines des nourrissons », il ressort des études portant sur la fiabilisation des systèmes complexes, que c'est un domaine très vaste qui requiert de vifs intérêts dans la plupart des domaines d'activités. Dès lors, pour contribuer à l'analyse et à l'optimisation de tels systèmes de plus en plus complexes tels que ceux de production de farines des nourrissons, nous avons défini une méthodologie de résolution de ce problème. Partant de l'analyse fonctionnelle, nous avons déterminé les fonctions de service et technologiques $\mathrm{du}$ broyeur à marteaux sur lequel nous nous sommes attardés et ensuite ressortit son diagramme F.A.S.T. De plus, l'optimisation de la fiabilité a été faite par le biais de la 《 conception robuste 》 couplée à un algorithme dans l'optique de définir une meilleure redondance pour notre unité de production.

Les résultats obtenus nous permettent de montrer que l'objectif initial a été atteint dans certaines proportions ; mais que des résultats plus édifiants peuvent cependant être obtenus en s'inspirant de ce travail réalisé.

En perspectives, il serait impératif à notre connaissance de : développer des modèles mathématiques d'optimisation de la fiabilité, prenant en compte des indicateurs de fiabilité.

\section{Références:}

1. Aftab A.S., and Styblinski N., (1994). IC variability minimization using a new $\mathrm{C}_{\mathrm{p}}$ and $\mathrm{C}_{\mathrm{pk}}$ based variability / performance measure. Proc. intern. Symp on Circuits and Systems (ISCAS), vol. 1: $149-152$.

2. Beleulmi S., Bellaouar A., chaib R., and Benidir M., (2011). Modeling the Reliability of a Mechanical System with Redundancy. Ouargla : 2 - 4.

3. Bhamare P., and Rathore Y., (2007) . Evolution of reliability engineering discipline over the last six decades: a comprehensive review. India. Journal of reliability and safety, vol .1: $337-410$.

4. Debyser A.G., and Gielen G., (1998). Efficient analog circuit synthesis with simultaneous yield and robustness optimization. California. Proc. intern. Conf : 308 - 311 on Computer - Aided Design (ICCAD).

5. Director S., Feldmann P., and Krishna K., (1992). Optimization of parametric yield : A tutorial. New York. Proceedings of the IEEE 1992 Custom Integrated Circuits Conference : 311 - 318.

6. Filiol H., (2010) . Methods for analyzing variability and robust design of analog circuits in advanced CMOS technologies. Grenoble. PhD thesis. Specialty: integrated electronics devices, 209 p. 
7. Gittard C., (2009) . Application of the system approach in the analysis of reliability during the upstream phases of the design of innovative products. Centrale Paris. Engineering memory, 45p.

8. Gondran M., Minoux M., Hery J.F., and Laleuf J.C., (1995) . Reliability of the systems. Paris. Collection of the direction of studies and research of electricity of France, Editions Eyrolles , 61. Bd Saint - Germain 5th : 251-254.

9. Keramat M., and Kielbasa R., (1997). Parametric yield optimization of electronic circuits via improved centers of gravity algorithm. Proceedings of the 40th Midwest Symposium on Circuits and Systems, vol. $2: 1415-1418$.

10. Mathelin S., (2006). Contribution to the improvement of collaborative design tools. Application to ergonomics in the automotive industry: an information system for integrating business perspectives into design. Grenoble. PhD thesis: INP, Laboratory GILCO (Industrial Management Logistics and Design), 176 p.

11. Mohammad A., (2016). A new model for reliability optimization of series -parallel systems with non - homogeneous components. Journal homepage: www.elsevier.com / locate / ress.

12. Mukherjee T., Carley L., and Rutenbar R., (2000) . Efficient handling of operating range and manufacturing line variations in analog cell synthesis. IEEE Transactions on Computer - Aided Design of Integrated Circuits and Systems, vol. 19, no. 8: 825 - 839.

13. Ozouf V., and Pillet M., (2006). Set up your AMDEC product through the APR. Centrale Paris.

14. Soin R. S., and Spence R., (1980). Statistical exploration approach to design centring. IEE Proceedings Electronic Circuits and Systems, vol. 127 , no. $6: 260-269$.

15. Zwingmann X., (2005). Model for assessing reliability and maintainability at the design stage. Quebec. Ph.D. thesis: Department of Mechanical Engineering, Faculty of Science and Engineering, Laval University, $379 \mathrm{p}$. 